\title{
A KNOWN PROBLEM OF GEOMETRY AND ITS CASES OF INDETERMINATION
}

\section{J. HADAMARD}

A recent note ${ }^{1}$ induced me to resume the classic problem $\left(\mathrm{P}_{0}\right)$ and its natural generalization:

$\left(\mathrm{P}_{0}\right)$ To construct a square, the sides of which go through four given points.

(P) To construct a quadrilateral $Q(X Y Z T)$ similar $^{2}$ to a given quadrilateral $q$ with its sides $X Y, Y Z, \cdots$, respectively passing through four given points $K, L, M, N$.

The solution of $\left(\mathrm{P}_{0}\right)$ is classic: we immediately get a locus for each vertex, namely the circumference having $K L, L M$ and so on for its diameter. Moreover, the diagonal $X Z$ of the required square must again cut the half circumference of diameter $N K$ at its middle point $\alpha$ and, similarly, the half circumference of diameter $L M$ at its middle point $\gamma$. This generally determines it, whence the required construction directly results.

The solution extends automatically to $(\mathrm{P})$ :

(i) We know a circular locus for each vertex of the unknown quadrilateral.

(ii) We also know the two points $\alpha, \gamma$ where the diagonal $X Z$ intersects the loci of the vertices $X, Z$.

This generally allows us to draw the required quadrilateral and:

(iii) The quadrilateral $Q$ constructed in that way is actually similar to $q$ and, therefore, satisfies all the conditions of the problem.

The verification of the latter fact offers no difficulty; but it must be insist ed on, for it is quite essential for what we are going to say. Indeed, instead of reasoning on the diagonal $X Z$, we could operate in the same way on the other diagonal $Y T$, of which, similarly, we know two points $\beta, \delta$, respectively belonging to the circumferences which are the loci of the vertices $Y$ and $T$; and it results from (iii) that this second construction necessarily gives the same result as the first one.

But, moreover, a singular case may occur, namely that in which

Received by the editors February 21, 1944.

1 Mathematicae Notae. Boletin del Instituto Matematica vol. 3 (1943) p. 155.

2 In the above-mentioned note, it is investigated whether the given points are on the sides themselves or on their prolongations, a question which we shall not examine.

On the other hand, for us, every similitude is meant to be a direct one. This is the reason why, from our point of view, the problem generally admits of one solution, while in the article in Mathematicae Notae it is considered as having two of them. 
the problem is indeterminate: $:^{3}$ this happens when $\alpha$ and $\gamma$ coincide. Now, if so, the same circumstance must appear when operating on the diagonal $Y T$ so that:

(iv) If $\alpha$ coincides with $\gamma$, then $\beta$ also coincides with $\delta$.

It is that remarkable circumstance which has seemed to me worth studying and, more generally, induced me to consider the relations between the points $\alpha, \beta, \gamma, \delta$.

I. In the special case of problem $\left(\mathrm{P}_{0}\right)$, everything can be settled by the properties and, especially, the rules of composition of rotations. Let us choose as unit angle the right angle in the direct sense (the retrograde right angle being denoted by -1 ). The triangles $\alpha N K$, and so on, being right-angled and isoceles, we have (the perimeter of our square being assumed to be described in the direct sense)

(1) (a) $K=\alpha_{1} N$

(b) $L=\beta_{1} K$,

(c) $M=\gamma_{1} L$,

(d) $N=\delta_{1} M$,

this meaning that $K$ results from $N$ by a direct rotation of a right angle around $\alpha$, and so on. Therefore, we also have

$$
L=\beta_{1} \alpha_{1} N,
$$

where the rotation $\omega_{2}=\beta_{1} \alpha_{1}$ is through the angle $180^{\circ}$, so that its center $\omega$ is the midpoint of $L N$. Moreover, the half-turn $\delta_{1} \gamma_{1}$ changes $N$ into $L$ and, therefore, is nothing else than $\beta_{1} \alpha_{1}$.

Now, the rules of composition of rotations show that $\omega$ is the vertex of a right-angled isosceles triangle, the hypotenuse of which is $\alpha \beta$, and similarly for $\gamma \delta$. We are here taking into account the senses of the angles,

$$
\beta=\omega_{-1} \alpha, \quad \delta=\omega_{-1} \gamma .
$$

This gives the answers to our questions:

(i) As the retrograde rotation $\omega_{-1}$, which is through a right angle, changes $\alpha$ into $\beta$ and $\gamma$ into $\delta$, we see that $\beta \delta$ is perpendicular to $\alpha \gamma$ and equal to $\alpha \gamma$.

(ii) The first part of this conclusion is evident on account of the fact that our problem is possible, as $\alpha \beta$ and $\gamma \delta$ are segments of the two diagonals of the square. But the question of senses deserves consideration. $\beta \delta$ is deduced from $\alpha \gamma$ by a rotation of -1 , while $b d$ is deduced from $a c$ by a rotation of +1 . Therefore, if we inquire whether $\alpha \gamma$ is of the same sense as $a c$, and whether $\beta \delta$ is of the same sense as $b d$,

${ }^{3}$ A single case of that kind has been mentioned, as far as we know: in the Nouvelles Annales de Mathématiques (see footnote 4), Laisant notices that indetermination takes place for a square circumscribed to a square. 
the two questions necessarily admit of contrary answers. If the diagram is such that $K, L, M, N$ lie on the sides of the square themselves $^{2}$ (and not on their continuations) and $\alpha, \beta, \gamma, \delta$ on the diagonals themselves, we can say that if the segments $a \alpha, c \gamma$ overlap, the segments $b \beta, d \delta$ do not, and conversely.

(iii) The equality $\beta \delta=\alpha \gamma$ shows us why $\delta$ must coincide with $\beta$ if $\gamma$ coincides with $\alpha$.

(iv) The condition for that is that the two lines $L N, K M$ be equal and perpendicular to each other (in other words, that the parallelogram whose vertices are the midpoints of the sides $K L, L M$, and so on, be a square), as the rotation $\alpha_{1}$ must change $N$ into $K$ and $L$ into $M$.

Similarly, both products $\gamma_{1} \beta_{1}$ and $\alpha_{1} \delta_{1}$ give a rotation of a half-turn around the midpoint $\pi$ of $K M$, so that

$$
\gamma=\pi_{-1} \beta, \quad \alpha=\pi_{-1} \delta .
$$

(v) The comparison of (3a) with (3) shows that the half-turn $\pi_{-1} \omega_{-1}$ takes place around the midpoint $C$ of $\alpha \gamma$ and the half-turn $\omega_{-1} \pi_{-1}$ around the midpoint $D$ of $\beta \delta$.

There are, therefore, two right-angled isosceles triangles having $C D$ for their common hypotenuse and $\omega, \pi$ for their vertices, located on both sides of $C D$. The midpoints of $K M, L N$ and the midpoints, $C, D$ of $\alpha \gamma, \beta \delta$ are the vertices of a square.

II. In order to treat the case where the given quadrilateral is no longer a square, but has an arbitrary shape, we must find the laws of composition not only of rotations, but also, more generally, of similitudes in the plane.4

When we shall speak of similitudes, it will be implicitly understood, if not otherwise specified, that we mean direct ones, in which not only the magnitudes but also the senses of the angles shall be preserved. In such a similitude, any line $M N$ in the second figure $F$ is in a constant ratio $r$ and makes a constant angle $\phi$ with the corresponding one $M_{0} N_{0}$ in the first figure $F_{0}$; this can be expressed algebraically by saying that the two complex numbers which represent $M N$ and $M_{0} N_{0}$ are in the constant complex ratio $\rho=r e^{i \phi}$.

If that ratio is equal to 1 (that is $r=1$ and $\phi=0$ ), the similitude reduces to a translation. In any other case, there is one and only one point $\alpha$-which we shall call the center of similitude-such that the

- A study of plane similitudes has been developed by Giusto Bellavitis, under the name of Theory of equipollences. It has been expounded in French in the Nouvelles Annales de Mathématiques vol. 8 (1869) (by Houël) and in a complete translation of Bellavitis' memoir in the same periodical, vol. 12 (1873), by Laisant. But the location of the new center in a product of two similitudes is not examined by Bellavitis. 
lines joining it to any point $M_{0}$ in $F_{0}$ and to its homologue $M$ in $F$ are represented by imaginaries the ratio of which is $\rho$. Thus, the triangle $\alpha M_{0} M$ will be similar to a fixed triangle $o a_{0} a$. Such transformations can be denoted by $\alpha_{(\phi, r)}$ (this differs from our former notation for rotations only by the introduction of the homothety ratio $r$, the angle $\phi$ being still measured with the direct right angle as unit) or by $\alpha_{\left(o a_{0} a\right)}$, the relation

$$
M=\alpha_{\left(o a_{0} a\right)} M_{0}
$$

meaning that the triangle $\alpha M_{0} M$ is directly similar to $o a_{0} a ; o a_{0} a$ can be called the characteristic triangle of the transformation.

Now we have to represent the product ${ }^{4}$ of two such similitudes $S_{0}, S_{1}$ with centers $\alpha, \beta$ and complex ratios $\rho_{0}=r_{0} e^{i \phi_{0}}, \rho_{1}=r_{1} e^{i \phi_{1}}$.

If the two centers coincide at $\alpha$, the answer is simple: the productsimilitude will have again $\alpha$ as its center, with a rotation angle $\phi_{0}+\phi_{1}$ and a similitude ratio $r_{0} r_{1}$. If we draw the two characteristic triangles so that they have one side $o a$ in common, the resultant characteristic triangle will consist of the two remaining sides $o a_{0}, o a_{1}$, and the connecting line $a_{0} a_{1}$.

The latter relation subsists in any case; but when the two centers do not coincide, we must find the center of the product-similitude $S_{1} S_{0}$.

We shall refer this general case to the first one by considering, as an intermediary, the composition of an arbitrary sinilitude $S_{0}=\alpha_{\left(o_{0} a\right)}$ with a translation. The latter will not alter the rotation angle $\phi_{0}$ nor the similitude ratio $r_{0}$, but there will be a new center $\alpha^{\prime}$ such that, $M_{0}$ being any point of the original figure, $M$ its transform by the given similitude, and $M^{\prime}$ the transform of $M$ by the translation, the triangle $\alpha^{\prime} M_{0} M^{\prime}$ will be similar to the characteristic triangle $o a_{0} a$ of $S_{0}$. Now, we immediately see that the segment $\alpha_{0} \alpha^{\prime}$ connecting this new center with the original one $\alpha$ can be deduced from $M_{0} M^{\prime}$, that is from the given translation, by a similitude the center of which is $M_{0}$ and the characteristic triangle the same as above, only with a different order of the vertices. Conversely, if a first similitude is given, another similitude with the same rotation angle and the same homothety ratio, but another center, can be considered as the product of the first one and a translation, the direction and magnitude of which are in the relation just obtained to the direction and magnitude of the line connecting the two centers.

We master the general case by simply combining the two above results. In order to obtain the product of the similitude $S_{0}=\alpha_{\left(o a_{0} a\right)}$ followed by the similitude $S_{1}=\beta_{\left(o a a_{1}\right)}$ (the two characteristic triangles being located so as to have the common side $o a$ ), we begin by intro- 
ducing the intermediary operation $S^{\prime}$ equipollent with $S_{1}$ (that is, having the same characteristic triangle) and homocentric with $S_{0}$. The product $S^{\prime} S_{0}$ is $\alpha_{\left(o a_{0} a_{1}\right)}$.

$S_{1}$ is deduced from $S^{\prime}$ and, therefore, $S_{1} S_{0}$ from $S^{\prime} S_{0}$ by composition with a translation $T$, the direction and magnitude of which are determined by submitting $\alpha \beta$ to a rotation whose angle is equal, in magnitude and sign, to $\angle o a a_{1}$, and changing its size in the ratio $a a_{1} / a o$.

Now, let $\omega$ be the center of the product-similitude. The translation $T$ is the same one which corresponds to changing $S^{\prime} S_{0}$ into the equipollent operation $S_{1} S_{0}$. Therefore; the imaginary quantity which represents the segment $\alpha \omega$ will be given by the relation

$$
(\alpha \omega)=\lambda(T),
$$

where the complex coefficient $\lambda$ is

$$
\lambda=\left(a_{0} 0\right) /\left(a_{0} a_{1}\right)
$$

(every segment within parentheses denoting the corresponding complex quantity). In a purely geometrical and real language, the segment $\alpha \omega$ will have the magnitude $|\lambda||T|$, with

$$
|\lambda|=\overline{a_{0} 0} / \overline{a_{1} a_{1}},
$$

and the direction making with $T$ the angle

$$
\psi=\angle o a_{0} a_{1} \text {. }
$$

Now, the form of the quantity (4) (taking into account the expression of $T$, a complex cross ratio) or, which is equivalent, the formulae (4a), (4b) suggest a rather unexpected, but unavoidable, connection with inversion. Let $\boldsymbol{a}_{0}, \boldsymbol{a}, \boldsymbol{a}_{1}$ be the inverse points of $a_{0}, a, a_{1}$ with respect to $o$ as pole: the triangle $\alpha \omega \beta$ will be inversely similar to $a_{1} a a_{0}$. It can be deduced from a triangle constructed on $\alpha \beta$ and directly similar to $a_{0} a a_{1}$ by a symmetry with respect to the axis of $\alpha \beta$.

III. The case of a lozenge can be investigated, with the exception of one single result, with the help of rotations only. Let $2 \theta$ and $2 \theta^{\prime}=2-2 \theta$ be the angles of the lozenge, still measured in right angles; the points $K, L, M, N$ will be deduced from $N, K, L, M$ by successive rotations around $\alpha, \beta, \gamma, \delta$, the angles of which will be alternately $2 \theta$ and $2 \theta^{\prime}$. Therefore, formulae (1a)-(1d) are to be replaced by

$$
K=\alpha_{2 \theta} N, \quad L=\beta_{2 \theta} K, \quad M=\gamma_{2 \theta} L, \quad N=\delta_{2 \theta} M .
$$

As the two angles of the lozenge are supplementary, we shall have

$$
L=\beta_{2 \theta^{\prime}} \alpha_{2 \theta} N=\omega_{2} N, \quad N=\delta_{2 \theta} \gamma_{2 \theta} L=\omega_{2} L,
$$


$\omega$ being again the midpoint of $L N$ and, therefore,

$$
\beta=\omega_{(-1, r)} \alpha, \quad \delta=\omega_{(-1, r)} \gamma,
$$

so that the segments $\alpha \gamma, \beta \delta$ must have perpendicular directions (with the same circumstance as above concerning senses) and a ratio between them equal to $r=\tan \theta=\overline{x z} / \overline{y t}$.

The condition for indetermination is that the lines $K M, L N$ be equal to each other and intersect at an angle equal to one of the angles of the lozenge.

The only essential difference from the above case is that the triangles $\omega \alpha \beta, \omega \gamma \delta, \pi \beta \gamma, \pi \delta \alpha$ are no longer isosceles. We must introduce the similitude $\Sigma$ obtained by the rotation -1 around $\omega$, combined with a homothety of ratio $r$, with respect to the same point as well as the analogous operation $\Sigma^{\prime}$, one of which changes $\alpha$ into $\beta$ and $\gamma$ into $\delta$, the other changing $\beta$ into $\gamma$ and $\delta$ into $\alpha$. It results therefrom that the midpoints $C, D$ of $\alpha \gamma, \beta \delta$ and the midpoints $\omega, \pi$ of $K M, L N$ are the vertices of a quadrilateral (a so-called "rhomboid") with two right angles, which has $C D$ as an axis of symmetry, the latter dividing it into two right-angled triangles similar to those into which the lozenge is divided by both its diagonals, while each of the triangles $C \omega \pi, D \omega \pi$ is similar to one of the halves into which the lozenge is divided by a single diagonal.

IV. Let us now take the general case of an arbitrary quadrilateral $q$. The successive similitudes which transform $N$ into $K$, and so on, have their centers at $\alpha, \beta, \gamma, \delta$; their characteristic triangles can be obtained in the following way. Let ${ }^{5}$ the diagonals $x z, y t$ of $q$ intersect at $o$ : if, from $o$, we draw $o k, o l, o m$, on perpendicular to the sides of $q$, onk, okl, and so on, will be the characteristic triangles.

We see that if these four similitudes were homocentric, their product would be the identical transformation. If they had different centers, the product would, as a rule, become a translation. But, in our case, there is at least one point which finally remains unchanged, namely $N$ : therefore, this translation must be zero and the product of the four similitudes

$$
S=\alpha_{(o n k)}, \quad S^{\prime}=\beta_{(o k l)}, \quad S^{\prime \prime}=\gamma_{(o l m)}, \quad S^{\prime \prime \prime}=\delta_{(o m n)}
$$

must again be the identical transformation.

The operation $S^{\prime \prime \prime} S^{\prime \prime}$ is the same as the operation $S^{-1} S^{\prime-1}$ : it changes $L$ into $N$ and its characteristic triangle is oln. We can find

' An exceptional case, which ought to be examined separately, would occur if the diagonals of $q$ were parallel, a circumstance which is possible if $q$, instead of being a proper quadrilateral, were a self-crossing four-sided closed line. 
its center, and even in two different ways, by our rule of composition. This introduces the inverse points $k, 1, \mathrm{~m}, \mathrm{n}$ of $k, l, m, n$ with respect to a circle having its center at $o$ : in other words, the poles of the sides of $q$ with respect to that circle. The connecting lines $k 1,1 \mathrm{~m}$, and so on, being the polar lines of the vertices $x, y, z, t$, our four inverse points are the vertices of a parallelogram..$^{\circ}$

The triangle $k 1 n$ is equal to $m n l$; the two triangles $\omega \alpha \beta, \omega \gamma \delta$ are directly similar to each other and inversely similar to the triangle $k \ln$. Therefore, the segments $\alpha \gamma, \beta \delta$ are in the ratio $k l / k n$, which is equal to $x z / y t$ (denoting by $x, y, z, t$ the inverse points of $x, y, z, t$ ) $=(x z / y t) \cdot(o y \cdot o t / o x \cdot o z)$.

As to the angle between these segments, it is, as is evident a priori, the angle between the diagonals of $q$. We can see, at least when $q$ is assumed to be convex, ${ }^{7}$ that the question of senses is to be answered as previously: for, in that case, the angle $\angle n k l$ is supplementary to $\angle x o y$.

The product $S S^{\prime \prime \prime}$ is the same as $S^{\prime-1} S^{\prime \prime-1}$ : its characteristic triangle is $o k m$; its center $\pi$ is such that each of the triangles $\pi \beta \gamma, \pi \delta \alpha$ is inversely similar to $\mathbf{n k m}$.



The product of the similitude $\Sigma=\omega_{(m n l)}$ followed by $\Sigma^{\prime}=\pi_{(n k m)}$ transforms $\alpha$ into $\gamma$, and the product of the same factors with reversal of the order changes $\beta$ into $\delta$. Each of them is a half-turn, as is seen by assembling the triangle $m n l$ with a triangle $m l^{\prime}$ equal to $n \mathrm{~km}$, so that their centers will be the midpoints $C, D$ of $\alpha \gamma, \beta \delta$.

- In the case of a lozenge, $\mathrm{klm}$ nould be a rectangle, which is decomposed by both its diagonals into four triangles, similar two by two to one-half of the lozenge.

7 This condition is not satisfied in the problem which is the object of the article in Mathematicae Notae, in which $q$ is formed by four consecutive sides of a regular polygon or their prolongations: such a quadrilateral is, of course, concave, except for a square or a regular pentagon. At least, matters are such if the sides are taken in their original order, which is not necessary (see footnote 9). 
The relations of these centers with $\omega, \pi$ are given by our rule of composition; having transferred the triangle $n k m$ into a position $\mathrm{mln}^{\prime}$ adjacent to $\mathrm{mln}$ along $\mathrm{ml}$ (see the figure), the triangle $C \omega \pi$ must be inversely similar to the triangle $\lambda \nu \nu^{\prime}$ the vertices of which are the inverse points of $1, n, n^{\prime}$ with respect to $m$, that is, as is easily seen, to the triangle formed by $k n$ and the two diagonals of the parallelogram $k 1 \mathrm{mn}$. We shall see, in the same way, that the midpoint $D$ of $\beta \delta$ forms with $\omega, \pi$ a triangle inversely similar to the one the sides of which are $k l$ and the two diagonals, so that the points $C, D, \omega, \pi$ are on the same circle and divide it harmonically. The point $I$ of intersection of the diagonals $X Z, Y T$ also belongs to that circumference. ${ }^{8}$

When $q$ is itself a parallelogram, the condition for the coincidence of $\alpha$ with $\gamma$ can be easily found and proves to be the same as that which implies the coincidence of $\beta$ with $\delta$; for in this case the similitude $S^{\prime \prime}$ will be the same as $S$, whence it immediately follows that the segments $K M, L N$ must intersect at the same angle and be in the same ratio as the sides of the given parallelogram; in other words, the midpoints of $K L, L M$, and so on, must be the vertices of a parallelogram similar to $q$.

In the general case the question is more complicated. The fact that $\mathrm{klmn}$ is a parallelogram is again essentially needed for its solution. Let $o k l, o m n$ be two triangles assumed, for simplicity's sake, to be entirely exterior to each other except for the common vertex 0 . Let us consider the replacement of the second triangle omn by any (directly) similar one $o m_{1} n_{1}$ having still a vertex at $o$, the other triangle $o k l$ remaining fixed. Among the transformations of this kind, there is precisely one such that the inverse points of the four vertices with respect to the pole $o$, taken in their due order, form a parallelogram; when this condition is satisfied, we can say that we have the reduced form of the diagram.

${ }^{8}$ That $I, C, D, \omega, \pi$ are concyclic can be seen in a more direct way: for any circle through $\omega, I$ divides $\alpha \gamma$ and $\beta \delta$ similarly-an elementary consequence of the direct similitude of $\omega \alpha \beta, \omega \gamma \delta$; and so does any circle through $\pi$ and $I$, but with an exchange between the roles of $\beta$ and $\delta$. It is clear that both correspondences agree when and only when the points of division are the midpoints.

In the case alluded to in footnote 5 , the triangles onk, okl, olm, omn can be obtained by considering any segment $o x$ parallel to the common direction of the diagonals of $q$ and drawing from $x$ parallels to the four sides of the polygonal line; $o k, o l$, $o m$, on will be perpendiculars dropped from $o$ on the lines thus drawn. The inverse points $k, 1, m, n$ will all lie on one straight line perpendicular to $o x$, so that $\omega$ will be the intersection between $\alpha \beta$ and $\gamma \delta, \pi$ the intersection of $\beta \gamma$ and $\delta \alpha$. Then $C D \omega \pi$ will be a straight line, divided harmonically by the two diagonals. As to the ratio $\beta \delta / \alpha \gamma=k 1 / k n$, it is easy to see that it will be equal to $x z / y t$. 
In the contrary case, let the original diagram oklmn be arbitrary. Applying a transformation of the aforesaid kind which changes $m, n$ into $m_{1}, n_{1}$, let us consider the two intermediate triangles olm, onk, which are changed into $o l m_{1}, o n_{1} k$. On the basis $l m_{1}$, we can construct a triangle $l m_{1} o_{1}$ directly similar to $l m o$ and, on the basis $n_{1} k$, the triangle $n_{1} k o_{1}^{\prime}$ directly similar to $n k o$; in general, the two new vertices $o_{1}, o_{1}^{\prime}$ will be different. They will coincide if the original diagram is a reduced one. ${ }^{9}$

New York Crty

9 The problem would require further investigation because there is no reason not to consider the complete quadrilateral formed by the sides of $q$ and, therefore, we ought to apply the present considerations to three diagonals instead of two.

\section{NOTE ON CONVEX SPHERICAL CURVES}

\section{A. SANTALó}

1. Introduction. The formula

$$
L=\int_{0}^{\pi} \alpha d \tau
$$

for plane convex curves in which $L$ is the length and $\alpha$ the breadth according to the direction $\tau$ is well known [2, p. 65].1

The principal object of the present note is to obtain the formula (8) which generalizes (1) to convex curves on the sphere of unit radius and to deduce from this some consequences.

2. Principal formula. Let us consider the sphere of unit radius. A closed curve on the sphere is said to be convex when it cannot be cut by a great circle in more than two points. It is well known that a convex curve divides the surface of the sphere into two parts, one of which is always wholly contained in a hemisphere; that is, there is always a great circle which has the whole convex curve on the same side. When we say the area of a convex curve $K$ we understand the area of that part of the surface of the sphere which is bounded by $K$ and is smaller than or equal to a hemisphere.

Let $K$ be a convex curve on the sphere of unit radius of length $L$ and area $F(L \leqq 2 \pi, F \leqq 2 \pi)$. The great circles which have only one common point or include a complete segment common with the curve

Received by the editors January 21, 1944.

1 Numbers in brackets refer to the references cited at the end of the paper. 